\title{
Insulin Adsorption to Catheter Materials Used for Intensive Insulin Therapy in Critically Ill Patients: Polyethylene Versus Polyurethane - Possible Cause of Variation in Glucose Control? ${ }^{\S}$
}

\author{
Stephan Christoph Ley ${ }^{*}, 1$, Jan Ammann ${ }^{2}$, Christian Herder ${ }^{3}$, Thorsten Dickhaus ${ }^{4}$, Matthias Hartmann ${ }^{5}$ \\ and Detlef Kindgen-Milles ${ }^{6}$
}

\author{
${ }^{I}$ Department of Anesthesiology and Intensive Care, St. Vinzenz Hospital Dinslaken, Germany \\ ${ }^{2}$ Department of Anesthesiology and Intensive Care, Slingeland Hospital Doetinchem, The Netherlands \\ ${ }^{3}$ Institute for Clinical Diabetology, German Diabetes Center, Duesseldorf, Germany \\ ${ }^{4}$ Weierstrass Institute for Applied Analysis and Stochastics, Berlin, Germany \\ ${ }^{5}$ Department of Anesthesiology and Intensive Care, University Hospital Essen, Germany \\ ${ }^{6}$ Department of Anesthesiology, University Hospital Duesseldorf, Germany
}

\begin{abstract}
Introduction: Restoring and maintaining normoglycemia by intensified insulin therapy in critically ill patients is a matter of ongoing debate since the risk of hypoglycemia may outweigh positive effects on morbidity and mortality. In this context, adsorption of insulin to different catheter materials may contribute to instability of glucose control. We studied the adsorption of insulin to different tubing materials in vitro and the effects on glycemic control in vivo.

Materials and Methods: In vitro experiments: A syringe pump was filled with $50 \mathrm{IU}$ insulin diluted to $50 \mathrm{ml}$ saline. A flow of $2 \mathrm{ml} / \mathrm{h}$ was perfused through polyethylene (PET) or polyurethane (PUR) tubing. Insulin concentrations were measured at the end of the tube for 24 hours using Bradford's protein assay. In vivo study: In a randomized doubleblinded cross-over design, 10 intensive care patients received insulin via PET and PUR tubes for 24 hours each, targeting blood glucose levels of $80-150 \mathrm{mg} / \mathrm{dl}$. We measured blood glucose levels, the insulin dose required to maintain target levels, and serum insulin and C-peptide levels.

Results: In vitro experiments: After the start of the insulin infusion, only 20\% (median, IQR 20-27) (PET) and 22\% (IQR 16-27) (PUR) of the prepared insulin concentration were measured at the end of the 2 meter tubing. Using PET, after one hour infusion the concentration increased to 34\% (IQR 29-36) and did not increase significantly during the next 24 hours (39\% (IQR 39-40)). Using PUR, higher concentrations were detected than for PET at every measurement from 1 hour (82\% (IQR 70-86)) to 24 hours (79\% (IQR 64-87)). In vivo study: Glycemic control was effective and not different between groups. Significantly higher volumes of insulin solution had to be infused with PET compared to PUR (median PET 70.0 (IQR 56-82) ml vs. PUR 42 (IQR 31-63) ml; p=0.0015). Serum insulin concentrations did not decrease significantly one hour after changing to PET or PUR tubing.

Conclusion: Polyurethane tubing systems allow application of insulin with significantly lower adsorption rates than polyethylene tubing systems. As a consequence, less insulin solution has to be infused to patients for effective blood glucose control. Tubing material of the insulin infusion may be crucial for safe and effective glycemic control in critically ill patients.
\end{abstract}

Keywords: Adsorption, critical illness, hypoglycemia, insulin, polyethylene, polyurethane.

\section{INTRODUCTION}

Restoring and maintaining normoglycemia in critically ill patients on medical and surgical intensive care units reduces

*Address correspondence to this author at the Department of Anesthesiology and Intensive Care, St. Vinzenz Hospital, Dr. Otto-SeidelStrasse 31-33, D-46535 Dinslaken, Germany; Tel: +45/2064/441152;

Fax: +45/2064/441157; Email: stephanley@o2online.de

${ }^{\S}$ The study was performed at the surgical intensive care unit of the University Hospital Duesseldorf, Heinrich-Heine-University, Duesseldorf, Germany. rates of infection, sepsis, acute renal failure and critical illness polyneuropathy [1-6]. As a consequence, morbidity, mortality, and length of stay in the ICU are reduced which is beneficial for patients and decreases overall treatment costs [7]. However, tight glycemic control might be associated with an increased risk of hypoglycemia [8]. In this context the premature termination of the Glucontrol and VISEP studies because of high rates of hypoglycemia have fuelled an ongoing debate [9].

At present, the causes leading to these high rates of hypoglycemia are not well defined and it is not clear whether 
and to what extent hypoglycemia harmed patients $[10,11]$. Most important, however, is the question why despite tight protocols hypoglycemia occurred so frequently. Some risk factors for low blood glucose levels during intensive insulin therapy (IIT) have been defined. Vriesendorp et al. showed bicarbonate-based substitution in hemofiltration, decrease of nutrition without adjustment of insulin infusion, a history of diabetes mellitus, sepsis and the use of inotropic drugs to be risk factors [12].

In the present discussion on intensified insulin protocols and hypoglycemia one important risk factor for unsteady insulin dosage did not receive appropriate attention yet: syringes and the plastic tubing used for insulin infusion were found to adsorb a considerable amount of insulin [13-17]. Some attempts have been made to reduce adsorption by adding blood [18], human albumin or gelatine [16] to the insulin solution. In fact, adsorption was decreased, but at least blood and human albumin are no longer used because of the risk of infection, and increased cost, respectively. Nevertheless, a major prerequisite for current protocols to keep blood glucose in a near-normal range is a constant and predictable insulin application over the whole range of doses required, which in some cases may well exceed 20 units of insulin per hour for some time [1]. In clinical practice, insulin doses are calculated from syringe pump infusion rates and the defined concentration of insulin in these systems. In fact, it was never investigated how much insulin finally reached the patient and what amount was adsorbed to catheter material.

The aims of the study were:

1. to compare, in vitro, adsorption of insulin onto two commonly used syringe and tubing systems (PET \& PUR)

2. to assess the effect of the two syringe and tubing systems on glycemic control in stable, critically ill patients.

\section{MATERIALS AND METHODS}

\section{In Vitro Study}

We studied a typical insulin syringe-catheter system used at the surgical intensive care unit (SICU) of the University Hospital Duesseldorf under laboratory conditions. A $50 \mathrm{ml}$ syringe consisting of polyethylene (Perfusor ${ }^{\circledR}$, BBraunMelsungen AG, Melsungen, Germany) was connected to a tubing system of polyethylene-ether (PET) (Original Perfusorleitung $^{\circledR}, 1.0 \times 2.0 \mathrm{~mm}$ diameter, $200 \mathrm{~cm}$ length, BBraunMelsungen AG) or polyurethane (PUR) (Verbindungsleitung $1 \times 2.35,200 \mathrm{~cm}$, BBraunMelsungen AG) ( $n=8$ for PUR, $n=6$ for PET). The syringe was filled with 50 IU of short-acting insulin (Actrapid ${ }^{\circledR}$, Novo Nordisk Pharma GmbH, Mainz, Germany) diluted with $47.5 \mathrm{ml}$ saline $0.9 \%$ to a concentration of 1 IU per $\mathrm{ml}$. A continuous infusion was started with $2 \mathrm{ml}$ per hour using an infusion pump (BBraunperfusor, BBraunMelsungen, Germany). During the first 5 hours aliquots were taken every hour at the end of the tubing. A final sample was taken 24 hours after the start of the infusion. In addition, we took samples from each syringe at the end of the experiments to measure insulin concentration in the syringe. Insulin concentration was measured by Bradford's protein assay [19] at a wavelength of $595 \mathrm{~nm}$ using a photometer ( $\mu$ Quant ${ }^{\mathrm{TM}}$ Microplate Spektralphotometer, Bio-Tek, Winooski, Vermont, USA).

\section{In Vivo Study}

The second part of the study was a randomized, doubleblinded cross-over trial in 10 patients at the SICU of the University Hospital Duesseldorf. The trial was approved by the ethics committee of the University (study number 2654), and written informed consent was obtained from the study participants or their legal representatives. Inclusion criteria were: expected length of stay in the ICU $>5$ days, stable hemodynamics and continuous external insulin requirement for 48 hours, age $>18$ years, no hemodialysis, no scheduled surgery in the intervention period, no cortisol medication. Each patient received insulin (Actrapid $^{\mathbb{B}} 2 \mathrm{IU} / \mathrm{ml}$ diluted with saline $0.9 \%$ ) for 24 hours via polyethylene and for another 24 hours via polyurethane tubing in a double-blinded manner at random order by a single central venous catheter lumen that was used only for these insulin infusions. Nursing staff were trained to maintain blood glucose between 80 and $150 \mathrm{mg} / \mathrm{dl}$ by adapting infusion rates of the syringe pump. Every 2-4 hours blood glucose was measured via a blood gas analyzer (ABL 700 or 800, Radiometer, Copenhagen, Denmark). Blood glucose values, infusion rates and additional insulin boluses were recorded as well as concomitant medications, fluid balance and the Therapeutic Intervention Scoring System (TISS-28) [20] and Simplified Acute Physiology Score (SAPS II) [21] scores. Two patients were excluded from the study because of need of acute hemodialysis (1 patient) and emergency surgery (1 patient).

Blood samples were obtained one hour before and one hour after the tubing was changed. Serum samples were analyzed for insulin and C-peptide concentration by ELISA (Insulin human ELISA and C-Peptide human ELISA, BIOSOURCETM Invitrogen Cytokines \&Signaling, BioSource Germany, Solingen, Germany). All results are shown as mean \pm standard deviation or median and interquartile range (IQR) as appropriate. Box plots show median and quartiles, lines illustrate $95 \%$ percentiles, and dots represent maximum and minimum values. Blood glucose was measured in $\mathrm{mg} / \mathrm{dl}$. Data were analyzed for significant differences using Wilcoxon rank tests as suitable. The statistical analysis software SAS version 9.2 (TS1M0), SAS Institute, Cary, NC, USA, was used for all analyses.

\section{RESULTS}

\section{In Vitro Study}

After start of the infusion, with both PET and PUR systems the insulin concentration detected at the end of the tubings was lower than the anticipated concentration, i.e. for PET only 20\% (median, IQR 20-27\%), and for PUR $22 \%$ (median, IQR 16-27 \%), respectively. With PET, the concentration rose to $34 \%$ (median, IQR 29-36\%) after one hour and did not increase substantially during the 24-hour study period (39\% (median, IQR 39-40 \%). With PUR, insulin concentrations were significantly higher at every measurement between 1 (81.5 (IQR 69.5-86) \%; p<0.05) and 24 hours (78.5 (IQR 63.5-87) \%; p<0.01) (Fig. 1). The insulin concentration measured in the syringes after 24 hours was on average $99 \%$ of the expected concentration. 


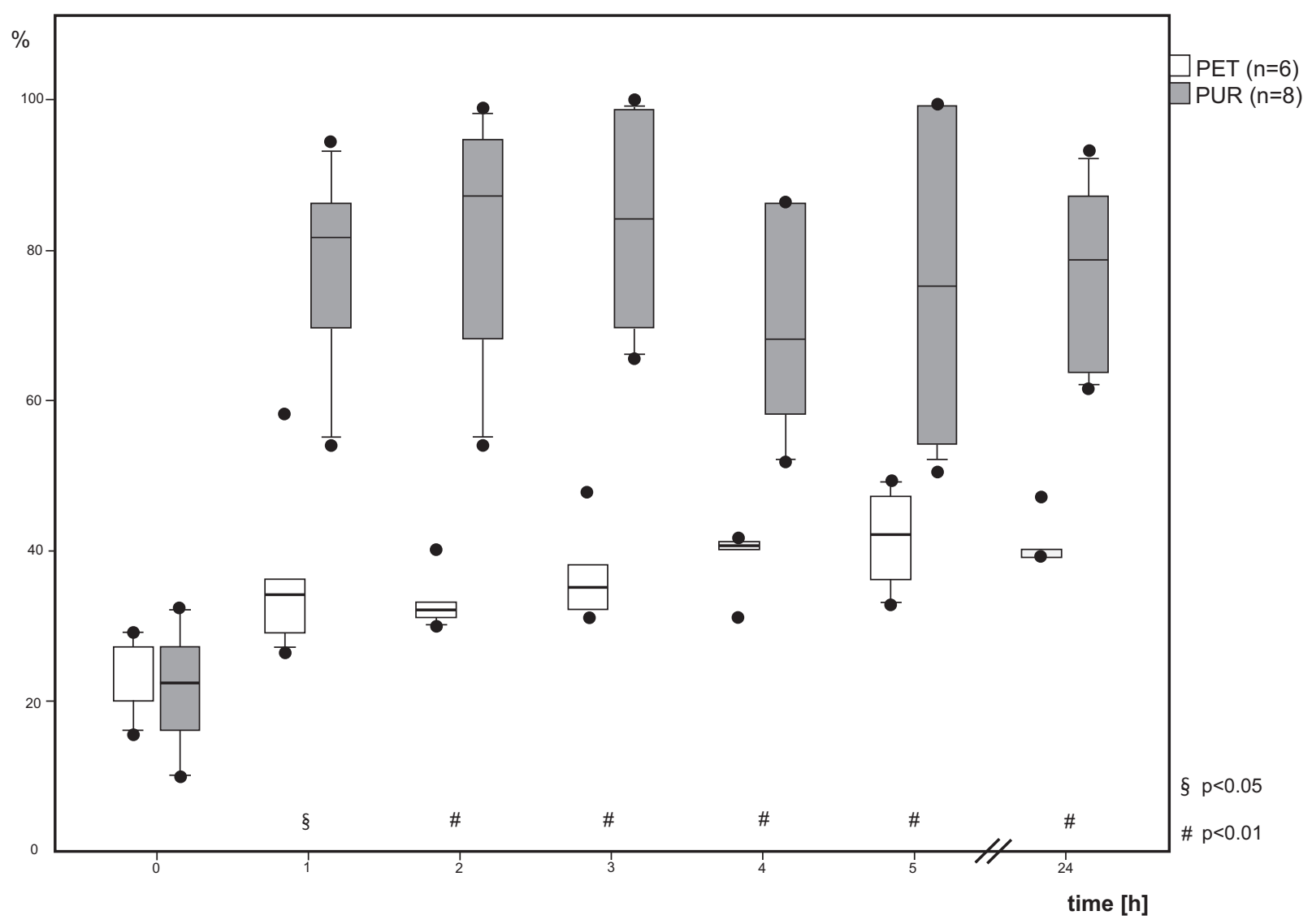

Fig. (1). Insulin concentration in $\%$ of expected concentration $(1 \mathrm{IU} / \mathrm{ml})$ at the end of PET or PUR infusion tubina.

\section{In Vivo Study}

The basic characteristics of the ten patients who participated in the in vivo part of the study are shown in Table 1. All patients required prolonged intensive care therapy following surgical procedures. All patients were critically ill, mechanically ventilated, and on catecholamine therapy. During the study period, there was no change in catecholamine dosage (for both norepinephrine and dobutamine, comparison of PET vs PUR: $\mathrm{p}=\mathrm{n} . \mathrm{s}$.), fluid balance $(\mathrm{p}=\mathrm{n} . \mathrm{s}$.) or severity scoring (TISS-28: $37 \pm 5$ (PET) vs $39 \pm 5$ (PUR); SAPS II: $42 \pm 14$ (PET) vs 41 \pm 16 (PUR); both n.s.).

Table 1. Baseline Characteristics of Patients.

\begin{tabular}{|c|c|c|c|c|c|c|c|}
\hline $\begin{array}{c}\text { Patient } \\
\text { No. }\end{array}$ & $\begin{array}{c}\text { Sex (m=Male, } \\
\mathbf{f}=\text { Female) }\end{array}$ & $\begin{array}{c}\text { Age } \\
\text { Years) }\end{array}$ & Admission Diagnosis & $\begin{array}{c}\text { Day on } \\
\text { ICU }\end{array}$ & $\begin{array}{c}\text { Diabetes } \\
\text { Diagnosis }\end{array}$ & $\begin{array}{c}\text { Fluid Balance } \\
\text { (PET/PUR-Day) }\end{array}$ & Renal Insufficiency \\
\hline \hline 1 & $\mathrm{~m}$ & 74 & Ischemic cerebral insult after CABG & 16 & DM type 2 & $+630 \mathrm{ml} /+875 \mathrm{ml}$ & compensated retention \\
\hline 2 & $\mathrm{~m}$ & 65 & Cardiogenic shock after CABG & 4 & none & $-2200 \mathrm{ml} /-2200 \mathrm{ml}$ & none \\
\hline 3 & $\mathrm{~m}$ & 81 & $\begin{array}{c}\text { Cardiogenic shock after aortic valve } \\
\text { replacement }\end{array}$ & 7 & none & $+1900 \mathrm{ml} /+2200 \mathrm{ml}$ & none \\
\hline 4 & $\mathrm{~m}$ & 49 & $\begin{array}{c}\text { Desobliteration of multiple arteries } \\
\text { due coral reef aorta }\end{array}$ & 15 & DM type 2 & $+1300 \mathrm{ml} /+2200 \mathrm{ml}$ & $\begin{array}{c}\text { Acute renal failure, but } \\
\text { no dialysis on study days }\end{array}$ \\
\hline 5 & $\mathrm{~m}$ & 71 & Cardiogenic shock after CABG & 11 & none & $+660 \mathrm{ml} / \pm 0 \mathrm{ml}$ & none \\
\hline 6 & $\mathrm{~m}$ & 64 & Cardogenic shock after CABG & 8 & DM type 2 & $\pm 0 \mathrm{ml} /+1000 \mathrm{ml}$ & none \\
\hline 7 & f & 69 & Polytrauma & 21 & none & $-100 \mathrm{ml} / \pm 0 \mathrm{ml}$ & none \\
\hline 8 & f & 69 & SIRS after CABG & 6 & DM type 2 & $-1990 \mathrm{ml} /+720 \mathrm{ml}$ & none \\
\hline 9 & m & 70 & GI-bleeding after CABG & 44 & none & $-844 \mathrm{ml} /+120 \mathrm{ml}$ & none \\
\hline 10 & m & 50 & Vertebralis-carotis-transposition & 5 & none & $-500 \mathrm{ml} /+2300 \mathrm{ml}$ & none \\
\hline
\end{tabular}


Glycemic control was similar between the intervention periods (Fig. 2). To maintain blood glucose within the target range, significantly higher volumes of insulin solution had to be infused with PET compared to PUR (PET 67 (IQR 56.2$81.5) \mathrm{ml}$ in $24 \mathrm{~h} v s$. PUR 44 (IQR 31-63) $\mathrm{ml}$ in 24h; $\mathrm{p}=0.0015)($ Fig. 3).

Insulin concentrations in the serum of our patients decreased from one hour before syringe/catheter change to one hour after the change from PUR to PET (median $-12.4 \%$ (IQR -4.1\%-(-) 37.3\%)) and increased from one hour before to one hour after change from PET to PUR (median 34.6\% (IQR $-13.9 \%-(+) 15.3 \%)$ ) without statistical significance $(\mathrm{p}=0.34)$. C-peptide as a marker of patients' own insulin production did not show significant differences before and after syringe/catheter change to PET/PUR (PET: $n=10$; median $0.46 \mathrm{pmol} / \mathrm{ml}$ (IQR 0.25-1.32); PUR: $\mathrm{n}=10$; median $0.71 \mathrm{pmol} / \mathrm{ml}$ (IQR 0.47-1.25); $\mathrm{p}=0.86)$.

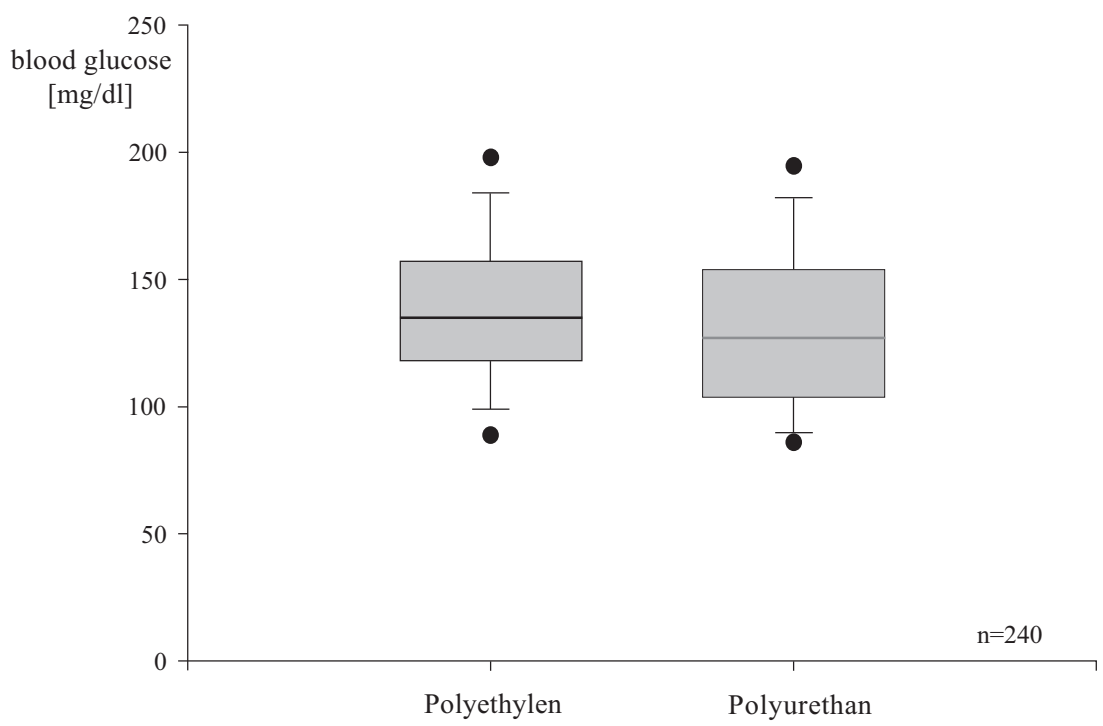

Fig. (2). There was no clinically relevant difference in quality of glucose control in days using polyurethane compared to polyethylene.

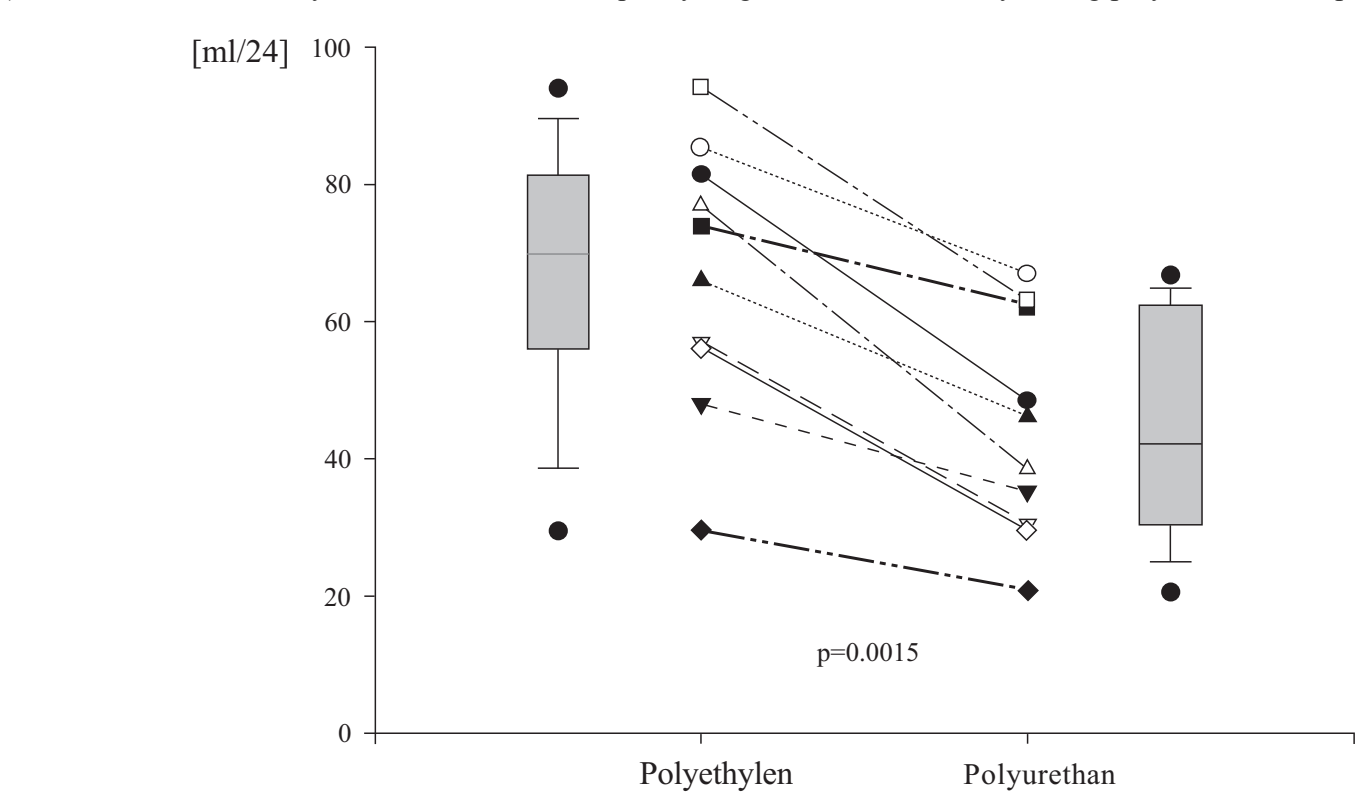

Fig. (3). Volume of infused insulin solution in 24 hours with polyethylene and polyurethane tubings. In every patient, less insulin solution

was infused when using polyurethane compared to polyethylene.

\section{DISCUSSION}

Tight glycemic control in critically ill patients has been shown to reduce morbidity and mortality $[1,2,6]$. In addition to improving outcome the intervention is also costeffective since the length of stay in the ICU is reduced [7]. In recent studies, however, these positive results have been questioned since frequent hypoglycemic episodes with potentially detrimental effects on outcome were observed. The mechanisms which may have caused hypoglycemia despite strict adherence to protocol-based adjustment of insulin infusion have been discussed at length [8].

Unfortunately, only little attention has been paid to the technical aspects of insulin administration in clinical practice. Although is well known that insulin is adsorbed to a variety of plastic materials, this aspect has not been evaluated in the context of intensified insulin therapy in critical care. Probably even more important than adsorption

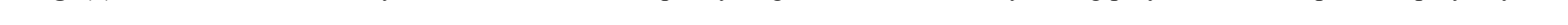


per se, however, is the variability of adsorption over time which we address here.

In the first clinical trial of van den Berghe [1] $10 \%$ of the patients required an infusion rate of more than $20 \mathrm{IU}$ of insulin per hour during the first 6 hours of therapy. The high amount of glucose infused during the investigation may have contributed to these high insulin doses. Unfortunately, no information was given with regard to the technical details of insulin application. Most likely, the material was polyethylene or polyvinylchloride because these materials are commonly used for catheters and syringes worldwide.

In our study, we detected significantly lower insulin concentrations than expected at the outlet of both tubing systems. Immediately following a syringe and line change only 20 (IQR 20-27) \% of the anticipated insulin dose left the infusion system in the PET arm of the in vitro trial, with an modest increase to $40 \%$ after 24 hours. In contrast, with PUR the delivered concentration rose to approximately $80 \%$ after one hour. In this way, significantly higher insulin doses are delivered at similar infusion rates.

The clinical relevance of these in vitro data was confirmed in our in vivo study. Overall control of blood glucose was not different between groups. However, with PET systems, patients required approximately 50\% more insulin solution compared to PUR systems to keep blood glucose within the targeted range. Thus, catheter material is crucial for the application of insulin to a patient, and large differences between the adsorption capacities of different catheter materials may have important clinical consequences. Our data clearly show that immediately following a change of the tubing system, the delivered insulin dose is not related to the infusion rate of the system. Thus, for a given time period, the actual insulin dose cannot be calculated from the infusion rate. In this setting, the delivered dose of insulin is lower than the prescribed dose which is in line with our observation, that the insulin concentrations in serum declined after a tubing change with both tubing materials one hour after the change. As a consequence, blood glucose may increase after a change of the infusion system which may cause staff to increase insulin infusion rate to restore blood glucose level. After a variable time, depending on the chosen concentration of insulin in the syringe and the infusion rate, the adsorption process is saturated and more insulin enters the systemic circulation of the patient. This timepoint may coincide with the effect of increasing the infusion rate and may, finally, promote hypoglycemia.

As a consequence of our data, more attention should be paid to the technical details of insulin application in critically ill patients, and one focus should be on syringe and tubing materials. From our data in vitro and in vivo, we can conclude that the catheter materials used today for continuous insulin infusion seem to be quite inappropriate for application of constant and predictable insulin doses. Comparing PET with PUR we showed that PUR - although slightly more expensive - had better, but also no ideal adsorption characteristics.

In addition to the effects caused by different tubing materials, adsorption might be influenced by the length of tubing and the concentration of insulin in the syringes. We only studied $2 \mathrm{~m}$ tubes because this is a standard length in our
ICU. Longer tubes may have more potent adsorbing properties and different time characteristics. Of note, the insulin concentration did not affect adsorption characteristics excessively. In preliminary experiments (data not shown) we found similar adsorption rates with insulin concentrations of 1 and $2 \mathrm{IU} / \mathrm{ml}$.

One of the limitations of this study is that we focused on PET and PUR only, and did not study other materials like polyvinylchloride (PVC). PVC has been shown to have a high adsorption potential as well and with PVC, different results might have been obtained. However, PVC is banned widely from European intensive care units and, therefore, we refrained from analyzing it. The number of experiments and patients in the in vivo part is small, however, the data obtained were very consistent. In every single patient a lower infusion volume was observed with PUR compared to PET. Thus, these observations should not be fundamentally different in a larger study population.

Finally, we have to discuss the course of the adsorbed insulin and potential interferences with confounding variables. This discussion is speculative, but insulin adsorption per se is a reversible process and we have to consider that a variety of other factors besides catheter materials may influence insulin availability. In our trial the insulin infusion was administered exclusively via a separate lumen of a central venous line. No other liquids or drugs were infused or injected via this lumen. In clinical practice, however, insulin is frequently infused in combination with other medications even if multi-lumen central venous catheters are available. If insulin is applied this way, variability of applied insulin doses may be increased for the following reasons. At first, varying flow rates in the system may per se influence the insulin administration. At second, the adsorption characteristics can differ with varying flow rates. At third, adsorption and release of insulin from the tubing material can be influenced by other drugs or medications, i.e. it cannot be excluded that other drugs may release insulin from the non-specific binding to catheter or tubing material. Adsorption to infusion tubings has been shown for a variety of drugs, i.e. nitroglycerin, clonazepam, amiodarone and others [22-24]. If these drugs interfere with insulin adsorption, unpredictable amounts of insulin could be released from their binding sites and reach the patient, this way promoting hypoglycemia.

Taken together, hypoglycemia in critically ill patients with tight glucose control occurs despite controlling well known risk factors for hypoglycaemia. Our data indicate that technical aspects like adsorption of insulin to tubing materials is important [12]. Thus, we most likely shall focus more on technical aspects of insulin application in critically ill patients. In addition, recent data suggest that not only severe hyperglycemia, but also a high variability of blood glucose level may increase mortality [5]. We have shown that the currently used catheter materials may contribute to a variation in insulin doses. If different materials are used in a single intensive care unit the doses can vary strongly. The effect of insulin adsorption to tubing systems and its effects on blood glucose control, therefore, must be taken into account when using intensified glucose control in the ICU. Intensivists should insist on infusion systems that deliver predictable amounts of insulin in time. 
Following our pilot study, further trials shall answer the question which factors beyond tubing materials influence insulin delivery. In this context, the use of different insulin concentrations in syringes, the use of different solvents, variations in flow rate through the catheter and interactions with other medications are of interest.

\section{CONCLUSION}

We have shown that polyurethane tubing systems adsorb significantly less insulin compared to polyethylene. As a clinical consequence, if polyurethane tubings are used, less insulin solution has to be infused to patients to obtain effective blood glucose control. Tubing material of the insulin infusion should be standardized to avoid variations in insulin delivery due to different adsorption characteristics. In general, insulin should be administered solely via a single (central) intravenous lumen. Improving the technical details of insulin application might be one important step to improve the safety of tight glycemic control in intensive care.

\section{CONFLICT OF INTEREST}

The authors confirm that this article content has no conflict of interest.

\section{ACKNOWLEDGEMENTS}

The authors thank the nurses and support personnel of the surgical intensive care unit of the University Hospital Duesseldorf for their support. We thank Ulrike Poschen (German Diabetes Center) for excellent technical assistance. Financial support came from an institutional grant (D. Kindgen-Milles).

\section{REFERENCES}

[1] van den Berghe G, Wouters P, Weekers F, et al. Intensive insulin therapy in the critically ill patients. N Engl J Med 2001; 19: 135967.

[2] van den Berghe G, Wilmer A, Hermans G, et al. Intensive insulin therapy in the medical ICU. N Engl J Med 2006; 5: 449-61.

[3] Krinsley JS. Effect of an intensive glucose management protocol on the mortality of critically ill adult patients. Mayo Clin Proc 2004; 8: 992-1000.

[4] Hermans G, Wilmer A, Meersseman W, et al. Impact of intensive insulin therapy on neuromuscular complications and ventilator dependency in the medical intensive care unit. Am J Respir Crit Care Med 2007; 5: 480-9.

[5] Egi M, Bellomo R, Stachowski E, French CJ, Hart G. Variability of blood glucose concentration and short-term mortality in critically ill patients. Anesthesiology 2006; 2: 244-52.
[6] Reed CC, Stewart RM, Sherman M, et al. Intensive insulin protocol improves glucose control and is associated with a reduction in intensive care unit mortality. J Am Coll Surg 2007; 5: 1048-54.

[7] van den Berghe G, Wouters PJ, Kesteloot K, Hilleman DE. Analysis of healthcare resource utilization with intensive insulin therapy in critically ill patients. Crit Care Med 2006; 3: 612-6.

[8] Krinsley JS, Grover A. Severe hypoglycemia in critically ill patients: risk factors and outcomes. Crit Care Med 2007; 10: 22627.

[9] Brunkhorst FM, Engel C, Bloos F, et al. Intensive insulin therapy and pentastarch resuscitation in severe sepsis. N Engl J Med 2008; 2: $125-39$.

[10] Ligtenberg JJ, Stemerdink A, Vogelzang M, Borggreve HF, Herngreen T, Zijlstra JG. Tight glucose control and hypoglycemia: should we bother? Crit Care Med 2007; 4: 1218.

[11] Vriesendorp TM, DeVries JH, van Santen S, et al. Evaluation of short-term consequences of hypoglycemia in an intensive care unit. Crit Care Med 2006; 11: 2714-8.

[12] Vriesendorp TM, van Santen S, DeVries JH, et al. Predisposing factors for hypoglycemia in the intensive care unit. Crit Care Med 2006; 1: 96-101.

[13] Schildt B, Ahlgren T, Berghem L, Wendt Y. Adsorption of insulin by infusion materials. Acta Anaesthesiol Scand 1978; 5: 556-62.

[14] Simeon PS, Geffner ME, Levin SR, Lindsey AM. Continuous insulin infusions in neonates: pharmacologic availability of insulin in intravenous solutions. J Pediatr 1994; 5: 818-20.

[15] Hewson M, Nawadra V, Oliver J, Odgers C, Plummer J, Simmer $\mathrm{K}$. Insulin infusions in the neonatal unit: delivery variation due to adsorption. J Paediatr Child Health 2000; 3: 216-20.

[16] Bohrer H, Krier C, Fleischer F, Jurs G, Schneider C. Adsorption of insulin to the Perfusor systems. Anasth Intensivther Notfallmed 1988; 1: 32-5.

[17] Fuloria M, Friedberg MA, DuRant RH, Aschner JL. Effect of flow rate and insulin priming on the recovery of insulin from microbore infusion tubing. Pediatrics 1998; 6: 1401-6.

[18] Kerchner J, Colaluca DM, Juhl RP. Effect of whole blood on insulin adsorption onto intravenous infusion systems. Am J Hosp Pharm 1980; 10: 1323-5.

[19] Bradford MM. A rapid and sensitive method for the quantitation of microgram quantities of protein utilizing the principle of proteindye binding. Anal Biochem 1976; 72: 248-54.

[20] Miranda DR, de Rijk A, Schaufeli W. Simplified Therapeutic Intervention Scoring System: the TISS-28 items--results from a multicenter study. Crit Care Med 1996; 1: 64-73.

[21] Le Gall JR, Lemeshow S, Saulnier F. A new Simplified Acute Physiology Score (SAPS II) based on a European/North American multicenter study. JAMA 1993; 270: 2957-63.

[22] Hansen HC, Spillum A. Loss of nitroglycerin during passage through two different infusion sets. Acta Pharm Nord 1991; 3: 1316.

[23] Schneider JJ, Good P, Ravenscroft PJ. Effect of tubing on loss of clonazepam administered by continuous subcutaneous infusion. J Pain Symptom Manage 2006; 6: 563-7.

[24] Weir SJ, Myers VA, Bengtson KD, Ueda CT. Sorption of amiodarone to polyvinyl chloride infusion bags and administration sets. Am J Hosp Pharm 1985; 12: 2679-83. 